Bulletin of the Section of Logic

Volume 46:1/2 (2017), pp. 75-91

http://dx.doi.org/10.18778/0138-0680.46.1.2.07

Wojciech Buszkowski

\title{
INVOLUTIVE NONASSOCIATIVE LAMBEK CALCULUS: SEQUENT SYSTEMS AND COMPLEXITY
}

\author{
Dedicated to Professor Grzegorz Malinowski
}

\begin{abstract}
In [5] we study Nonassociative Lambek Calculus (NL) augmented with De Morgan negation, satisfying the double negation and contraposition laws. This logic, introduced by de Grooté and Lamarche [10], is called Classical Non-Associative Lambek Calculus (CNL). Here we study a weaker logic InNL, i.e. NL with two involutive negations. We present a one-sided sequent system for InNL, admitting cut elimination. We also prove that InNL is PTIME.

Keywords: nonassociative Lambek calculus, linear logic, sequent system, cut elimination, PTIME complexity

\section{Introduction and preliminaries}

InNL is a nonassociative version of noncommutative multiplicative linear logic (noncommutative MLL) of Abrusci [1], but - like in CNL - the multiplicative (intensional) constants 1, 0 are not admitted. Nonetheless, all results remain true for InNL augmented with these constants, denoted by InNL1.

These logics belong to substructural logics [8]. Associative and commutative substructural logics include several important non-classical logics, e.g. many-valued and fuzzy logics [14]. Nonassociative substructural logics, usually some extensions of NL, are used in type grammars as logics of syntactic types of structured expressions [3].
\end{abstract}


InNL1 with additive (extensional) connectives $\wedge, \vee$ amounts to InGL (involutive groupoid logic) from Galatos and Jipsen [7]; it is a conservative extension of InNL1. [7] provides a two-sided sequent system for InGL, admitting cut elimination. By the subformula property, this system restricted to multiplicative connectives and constants is good for InNL1. We, however, present a simpler, one-sided sequent system. The system from [7] employs special structural operators for negations, and the corresponding structural rules reflect the laws of free involutive groupoids, which complicates formal proofs and meta-logical proofs (e.g. of decidability, finite model property). Our systems are better suited for these purposes.

The paper [10], introducing CNL, is purely proof-theoretic: the authors focus on proof nets. [5] defines phase spaces for CNL and employs them in proofs of some meta-logical theorems; phase spaces for InNL (denoted there by $\mathrm{CNL}^{-}$) are defined, but not worked out. Phase spaces for associative substructural logics were studied in $[9,1,15]$. The present paper is mostly proof-theoretic (but we avoid proof nets). The semantic notions are limited to some basic algebras. Phase spaces for InNL and its extensions have been elaborated in [6].

[10] proves that CNL is PTIME. The main technical result of the present paper establishes the PTIME complexity of InNL (in a different way). [5] shows that the finitary consequence relation for CNL is PTIME, like for NL [3]. Those methods, however, cannot be applied to InNL. The complexity of the consequence relation for InNL is left as an open problem.

Now, we briefly describe the algebraic models of our logics.

The algebraic models of NL are residuated groupoids, i.e. (ordered) algebras $\mathbf{M}=(M, \otimes, \backslash, /, \leq)$ such that $(M, \leq)$ is a poset, and $\otimes, \backslash, /$ are binary operations on $M$, satisfying the residuation laws:

$$
a \otimes b \leq c \text { iff } b \leq a \backslash c \text { iff } a \leq c / b
$$

for all $a, b, c \in M$. A residuated groupoid $\mathbf{M}$ is unital, if it contains an element 1 such that $1 \otimes a=a=a \otimes 1$, for any $a \in M$. For any residuated groupoid $\mathbf{M}$, the product $\otimes$ is isotone in both $\operatorname{arguments}$, hence $(M, \otimes, \leq)$ is a p.o. groupoid. We refer to $\backslash, /$ as the residual operations for product.

The algebraic models of InNL are involutive residuated groupoids, i.e. algebras $\mathbf{M}=(M, \otimes, \backslash, /, \sim,-, \leq)$ such that $(M, \otimes, \backslash, /, \leq)$ is a residuated groupoid, and $\sim,-$ are antitone (i.e. order-reversing) unary operations on $M$, satisfying the double negation laws and the contraposition law:

$$
\text { (DN) } a^{\sim^{-}}=a=a^{-\sim} \text {, }
$$




$$
(\mathrm{CON}) a^{\sim} / b=a \backslash b^{-}
$$

for all $a, b \in M$. One easily derives other contraposition laws: $a \backslash b=a^{\sim} / b^{\sim}$, $a / b=a^{-} \backslash b^{-}$. In a unital involutive residuated groupoid, there holds $1^{\sim}=1^{-}$, since $1^{\sim}=1^{\sim} / 1=1 \backslash 1^{-}=1^{-}$(in any unital residuated groupoid, $a / 1=a=1 \backslash a)$. One defines $0=1^{\sim}$.

One shows: $\left(b^{-} \otimes a^{-}\right)^{\sim}=\left(b^{\sim} \otimes a^{\sim}\right)^{-}$, for all $a, b$, and defines the dual product: $a \oplus b=\left(b^{-} \otimes a^{-}\right)^{\sim}$ (also called par in the literature on linear logics). If $1 \in M$, then $0 \oplus a=a=a \oplus 0$. One obtains: $a \backslash b=a^{\sim} \oplus b$, $a / b=a \oplus b^{-}$. Hence $a \backslash b=\left(b^{-} \otimes a\right)^{\sim}, a / b=\left(b \otimes a^{\sim}\right)^{-}$. In unital involutive residuated groupoids, there hold: $a^{\sim}=a \backslash 0, a^{-}=0 / a$.

Involutive residuated groupoids are term equivalent to algebras, called involutive p.o. groupoids in [8], i.e. $\left(M, \otimes,^{\sim},-, \leq\right)$ such that $(M, \otimes)$ is a groupoid, $(M, \leq)$ is a poset, and $\sim,-$ are antitone unary operations on $M$, satisfying $(\mathrm{DN})$ and the compatibility condition:

$(\mathrm{COMP})$ if $a \otimes b \leq c$ then $c^{-} \otimes a \leq b^{-}$and $b \otimes c^{\sim} \leq a^{\sim}$,

for all $a, b, c \in M$. (COMP) holds in any involutive residuated groupoid. In an involutive p.o. groupoid, one defines $\backslash, /$ in terms of $\otimes, \sim,-$ as above, which yields an involutive residuated groupoid. Notice that (COMP) entails the converse implications:

$$
\begin{aligned}
& \text { if } c^{-} \otimes a \leq b^{-} \text {then } a \otimes b \leq c, \\
& \text { if } b \otimes c^{\sim} \leq a^{\sim} \text { then } a \otimes b \leq c .
\end{aligned}
$$

An involutive residuated groupoid is said to be cyclic, if $a^{\sim}=a^{-}$, for any element $a$. Cyclic involutive residuated groupoids are algebraic models of CNL; see [5]. CNL might also be named Cyclic Involutive Nonassociative Lambek Calculus (CyInNL), following the terminology of [8].

Now, we present NL and InNL as intutionistic sequent systems.

The formulas of $\mathrm{NL}$ are built from variables $p, q, r, \ldots$ by means of connectives $\otimes, \backslash, /$. One defines bunches: (i) every formula is a bunch, (ii) if $\Gamma$ and $\Delta$ are bunches, then $(\Gamma, \Delta)$ is a bunch. Bunches can be treated as the elements of the free groupoid generated by the set of formulas. $N L$ sequents are of the form $\Gamma \Rightarrow A$, where $\Gamma$ is a bunch and $A$ is a formula. $A$ context is a bunch containing a special atom $x$ (a place for substitution). We denote formulas by $A, B, C, \ldots$, bunches by $\Gamma, \Delta, \Theta$, and contexts by $\Gamma[], \Delta[]$ etc. $\Gamma[\Delta]$ denotes the result of substituting $\Delta$ for $x$ in $\Gamma[]$. The axioms and the inference rules of NL are as follows. 


$$
\begin{gathered}
\text { (NL-id) } A \Rightarrow A \quad \text { (NL-cut) } \frac{\Gamma[A] \Rightarrow B \Delta \Rightarrow A}{\Gamma[\Delta] \Rightarrow B} \\
(\otimes \Rightarrow) \frac{\Gamma[(A, B)] \Rightarrow C}{\Gamma[A \otimes B] \Rightarrow C} \quad(\Rightarrow \otimes) \frac{\Gamma \Rightarrow A \Delta \Rightarrow B}{(\Gamma, \Delta) \Rightarrow A \otimes B} \\
(\backslash \Rightarrow) \frac{\Gamma[B] \Rightarrow C \Delta \Rightarrow A}{\Gamma[(\Delta, A \backslash B)] \Rightarrow C} \quad(\Rightarrow \backslash) \frac{(A, \Gamma) \Rightarrow B}{\Gamma \Rightarrow A \backslash B} \\
(/ \Rightarrow) \frac{\Gamma[A] \Rightarrow C \Delta \Rightarrow B}{\Gamma[(A / B, \Delta)] \Rightarrow C} \quad(\Rightarrow /) \frac{(\Gamma, B) \Rightarrow A}{\Gamma \Rightarrow A / B}
\end{gathered}
$$

This sequent system is due to Lambek [11]. NL1 is obtained by admitting the empty bunch $\epsilon$, satisfying $(\epsilon, \Gamma)=\Gamma=(\Gamma, \epsilon)$, and the constant 1 (an atom) with two new rules and one new axiom.

$$
(1 \Rightarrow) \frac{\Gamma[\Delta] \Rightarrow A}{\Gamma[(1, \Delta)] \Rightarrow A} \quad \frac{\Gamma[\Delta] \Rightarrow A}{\Gamma[(\Delta, 1)] \Rightarrow A} \quad(\Rightarrow 1) \epsilon \Rightarrow 1
$$

We write $\Rightarrow A$ for $\epsilon \Rightarrow A$. NL1 is not a conservative extension of NL; $p /(q / q) \Rightarrow p$ is provable in NL1 but not in NL. Lambek [11] proved the cut-elimination theorem: every sequent provable in NL is provable without (NL-cut). His standard syntactic proof can easily be adapted for NL1.

InNL (resp. InNL1) can be presented as an extension of NL (resp. NL1) with new unary connectives $\sim,-$, the new axioms:

$$
\text { (a.DN) } A^{\sim-} \Leftrightarrow A, A^{-\sim} \Leftrightarrow A \quad(\text { a.CON }) A^{\sim} / B \Leftrightarrow A \backslash B^{-},
$$

and the new inference rules:

$$
(\mathrm{r}-\mathrm{CON}) \frac{A \Rightarrow B}{B^{\sim} \Rightarrow A^{\sim}} \quad \frac{A \Rightarrow B}{B^{-} \Rightarrow A^{-}} .
$$

We write $A \Leftrightarrow B$ for ' $A \Rightarrow B$ and $B \Rightarrow A$ '. Notice that in InNL1 (r-CON) can be omitted; they are derivable in the resulting system.

CNL (resp. CNL1) can be obtained from InNL (resp. InNL1) by adding the axioms $A^{\sim} \Leftrightarrow A^{-}$. Equivalently, one omits ${ }^{-}$and replaces ${ }^{-}$by $\sim$ in the afore-mentioned axioms and rules.

Using standard tools (e.g. the Lindenbaum-Tarski algebra), one proves that NL is strongly complete with respect to residuated groupoids. This means that for any set of sequents $X$ and any sequent $\Gamma \Rightarrow A, X \vdash_{N L} \Gamma \Rightarrow$ 
$A$ if and only if, for any residuated groupoid $\mathbf{M}$ and any valuation $\mu$ in $\mathbf{M}$, if $\mathbf{M}, \mu \models X$ then $\mathbf{M}, \mu \models \Gamma \Rightarrow A$. Recall that $\mu$ is a homomorphism from the formula algebra to $\mathbf{M}$; it is extended for bunches by: $\mu((\Gamma, \Delta))=$ $\mu(\Gamma) \otimes \mu(\Delta) . \mathbf{M}, \mu \models \Gamma \Rightarrow A$ means: $\mu(\Gamma) \leq \mu(A)$.

Analogously, NL1 is strongly complete with respect to unital residuated groupoids. Now $\mu(1)=\mu(\epsilon)=1$. InNL (resp. CNL) is strongly complete with respect to (resp. cyclic) involutive residuated groupoids and InNL1 (resp. CNL1) with respect to the unital algebras from this class.

The systems for InNL, InNL1, CNL, CNL1, presented above, do not admit cut elimination. A (one-sided) cut-free system for CNL was given in [10]. This system is in Schütte style; the sequent $\Gamma$ means $\Rightarrow \Gamma$, and each comma in $\Gamma$ is interpreted as dual product. [5] studies a dual Schütte style system for CNL; the sequent $\Gamma$ means $\Gamma \Rightarrow$, and each comma in $\Gamma$ is interpreted as product. For (associative) bilinear logic, a system of the latter form was considered by Lambek [12].

In this paper, we present analogous systems for InNL and InNL1. We prefer dual Schütte style systems, because their syntax is closer to the intuitionistic systems NL, NL1, which facilitates some constructions and arguments. All results can be easily adapted for Schütte style systems.

[5] shows that CNL is a strongly conservative extension of NL. This means that for any set of NL-sequents $X$ and any NL-sequent $\Gamma \Rightarrow A$, $X \vdash_{N L} \Gamma \Rightarrow A$ if and only if $X \vdash_{C N L} \Gamma \Rightarrow A$. Also CNL1 is a strongly conservative extension of NL1. Since InNL (resp. InNL1) is intermediate between NL (resp. NL1) and CNL (resp. CNL1), InNL (resp. InNL1) is evidently a strongly conservative extension of NL (resp. NL1).

Let us note an algebraic characterization of strong conservativeness. A system $S^{\prime}$ is a strongly conservative extension of a system $S$ if and only if every algebraic model for $S^{\prime}$ is an expansion of a model for $S$ and every model for $S$ is embeddable in a model for $S^{\prime}$. This characterization has some value for applications. In mathematical linguistics, an intended model for NL is the powerset of the set of all finite (skeletal) binary trees over a finite alphabet; the trees are interpreted as phrase structures of expressions of a language. These algebras need not admit linguistically natural negation operations, satisfying $(\mathrm{DN}),(\mathrm{CON})$. They, however, can be isomorphically embedded in algebras with such operations. Therefore logics with negations of this kind can be used for natural language processing, e.g. in type grammars, instead of their intuitionistic fragments, with a sound interpretation in the extended models. 
We note a striking difference between CNL and InNL: the former possesses the strong finite model property, but the latter does not [5]. In involutive residuated groupoids (even involutive posets) $a^{\sim}<a^{-}$gives rise to the infinite chain $a<a^{2 \times \sim}<a^{4 \times \sim}<\ldots$, hence in finite models $p^{\sim} \Rightarrow p^{-}$ implies $p^{-} \Rightarrow p^{\sim}$. There exist infinite models with $a^{\sim}<a^{-}$for some elements $a$, e.g. Lambek's pregroup of all unbounded order-preserving maps on $\mathbb{Z}$ (it can be shown that $f^{\sim}<f^{-}$for every map $f$ which is injective but not surjective). Pregroups are special involutive residuated monoids, where $\otimes=\oplus$ and $1=0$. A model with $\otimes \neq \oplus$ can be constructed as an algebra of downsets of Lambek's pregroup [13]. In the literature on pregroups, one writes $a^{r}$ for $a^{\sim}$ and $a^{l}$ for $a^{-}$.

This paper is organized as follows. In section 2 we present sequent systems for InNL and InNL1; the cut-elimination theorem is proved in a standard (syntactic) way. Section 3 establishes the PTIME complexity of InNL (this also holds for InNL1).

\section{Sequent systems}

We present our one-sided sequent system for InNL. (Other systems have been proposed in [2]; they, however, do not possess the subformula property). Propositional variables are denoted by $p, q, r, \ldots$ Atomic formulas (atoms) are of the form $p^{(n)}$, where $p$ is a variable and $n \in \mathbb{Z}$. One interprets $p^{(n)}$, for $n \geq 0$, as $p^{\sim \cdots \sim}$ with $\sim$ occurring $n$ times, and $p^{(n)}$, for $n<0$, as $p^{-\cdots-}$ with ${ }^{-}$occurring $|n|$ times. The connectives are $\otimes, \oplus$.

Bunches are defined as for NL. The meta-logical notation is like for NL. Sequents are the bunches containing at least two formulas. Often we omit the outer parentheses in sequents.

The axioms of our cut-free system are:

(id) $p^{(n)}, p^{(n+1)}$ for all variables $p$ and $n \in \mathbb{Z}$.

The inference rules are as follows.

$$
\begin{gathered}
(\mathrm{r}-\otimes) \frac{\Gamma[(A, B)]}{\Gamma[A \otimes B]} \\
(\mathrm{r}-\oplus 1) \frac{\Gamma[B] \Delta, A}{\Gamma[(\Delta, A \oplus B)]} \quad(\mathrm{r}-\oplus 2) \frac{\Gamma[A] B, \Delta}{\Gamma[(A \oplus B, \Delta)]}
\end{gathered}
$$




$$
\text { (r-shift) } \frac{(\Gamma, \Delta), \Theta}{\overline{\Gamma,(\Delta, \Theta)}}
$$

The algebraic models are involutive residuated groupoids. Every valuation $\mu$ must satisfy: $\mu\left(p^{(n+1)}\right)=\mu\left(p^{(n)}\right)^{\sim}$, for any atom $p^{(n)}$; it follows that $\mu\left(p^{(n-1)}\right)=\mu\left(p^{(n)}\right)^{-} . \mu$ is extended for sequents by: $\mu((\Gamma, \Delta))=$ $\mu(\Gamma) \otimes \mu(\Delta)$. We define: $\mathbf{M}, \mu \models \Gamma, \Delta$ iff $\mu(\Gamma) \leq \mu(\Delta)^{-}$(equivalently: $\left.\mu(\Delta) \leq \mu(\Gamma)^{\sim}\right)$.

Observe that (r-shift), looking like a weak associativity rule, expresses the contraposition laws. In algebraic terms: $a \otimes b \leq c^{-}$iff $b \leq a \backslash c^{-}$iff $b \leq a^{\sim} / c$ iff $b \otimes c \leq a^{\sim}$.

We denote this system by S-InNL. Negations $\sim,-$ are defined in metalanguage.

$$
\begin{array}{cl}
\left(p^{(n)}\right)^{\sim}=p^{(n+1)} & \left(p^{(n)}\right)^{-}=p^{(n-1)} \\
(A \otimes B)^{\sim}=B^{\sim} \oplus A^{\sim} & (A \oplus B)^{\sim}=B^{\sim} \otimes A^{\sim} \\
(A \otimes B)^{-}=B^{-} \oplus A^{-} & (A \oplus B)^{-}=B^{-} \otimes A^{-}
\end{array}
$$

One easily proves $A^{\sim-}=A, A^{-\sim}=A$, by induction on $A$. If $\mu$ is a valuation in an involutive residuated groupoid, then a straightforward induction on $A$ yields:

$$
\mu\left(A^{\sim}\right)=\mu(A)^{\sim}, \mu\left(A^{-}\right)=\mu(A)^{-}, \text {for any formula } A .
$$

We write $\vdash \Gamma$, if $\Gamma$ is provable in S-InNL. By induction on derivations in $\mathrm{S}-\mathrm{InNL}$ one easily proves that $(\mathrm{r}-\otimes)$ is reversible: if $\vdash \Gamma[A \otimes B]$, then $\vdash \Gamma[(A, B)]$. The following lemma can be proved by induction on $A$.

Lemma $1 . \vdash A^{-}, A$ and $\vdash A, A^{\sim}$, for any formula $A$.

We want to prove that the cut rules:

$$
\left(\mathrm{cut}^{\sim}\right) \frac{\Gamma[A] \Delta, A^{\sim}}{\Gamma[\Delta]}\left(\mathrm{cut}^{-}\right) \frac{\Gamma[A] A^{-}, \Delta}{\Gamma[\Delta]}
$$

are admissible in S-InNL. With the cut-rules this system is strongly complete with respect to involutive residuated groupoids (see Theorem 2).

By $S_{0}$ we denote the system arising from S-InNL, after one has replaced (r-shift) with two new rules.

$$
(\mathrm{r}-\oplus 3) \frac{A, \Gamma B, \Delta}{A \oplus B,(\Delta, \Gamma)} \quad(\mathrm{r}-\oplus 4) \frac{\Gamma, A \Delta, B}{(\Delta, \Gamma), A \oplus B}
$$


$(\mathrm{r}-\oplus 3)$ is derivable in S-InNL, by $(\mathrm{r}-\oplus 2)$ and ( $\mathrm{r}-\mathrm{shift})$, and $(\mathrm{r}-\oplus 4)$ is derivable, by $(\mathrm{r}-\oplus 1)$ and (r-shift). By $\vdash_{0}$ we denote the provability in $S_{0}$.

Lemma 2. The rule ( $r$-shift) is admissible in $S_{0}$.

Proof: We only prove the admissibility of the top-down direction of (rshift): if $\vdash_{0}\left(\Gamma_{1}, \Gamma_{2}\right), \Gamma_{3}$ then $\vdash_{0} \Gamma_{1},\left(\Gamma_{2}, \Gamma_{3}\right)$. The converse implication is proved in a similar way. We proceed by induction on the proof of $\left(\Gamma_{1}, \Gamma_{2}\right), \Gamma_{3}$ in $S_{0}$. This sequent cannot be an axiom (id). Then, it must be the conclusion of a rule. We consider several cases.

$(\mathrm{r}-\otimes)$. Then, the active formula $A \otimes B$ occurs in $\Gamma_{i}$ for some $1 \leq i \leq 3$. Take $i=1$. So $\Gamma_{1}=\Delta[A \otimes B]$ and the premise is $\left(\Delta[(A, B)], \Gamma_{2}\right), \Gamma_{3}$. By the induction hypothesis, $\vdash_{0} \Delta[(A, B)],\left(\Gamma_{2}, \Gamma_{3}\right)$, hence $\vdash_{0} \Gamma_{1},\left(\Gamma_{2}, \Gamma_{3}\right)$, by $(\mathrm{r}-\otimes)$. For $2 \leq i \leq 3$, the argument is similar.

$(\mathrm{r}-\oplus 1)$. We consider two subcases. $1^{\circ}$. The active bunch $(\Delta, A \oplus B)$ occurs in $\Gamma_{i}$ for some $1 \leq i \leq 3$. We apply the induction hypothesis, as above. $2^{\circ} .(\Delta, A \oplus B)=\left(\Gamma_{1}, \Gamma_{2}\right)$. Then, $\Gamma_{1}=\Delta, \Gamma_{2}=A \oplus B$, and the premises are $B, \Gamma_{3}$ and $\Delta, A$. One derives $\Delta,\left(A \oplus B, \Gamma_{3}\right)$, by $(\mathrm{r}-\oplus 2)$.

$(\mathrm{r}-\oplus 2)$. We consider two subcases. $1^{\circ}$. The active bunch $(A \oplus B, \Delta)$ occurs in $\Gamma_{i}$ for some $1 \leq i \leq 3$. We apply the induction hypothesis, as above. $2^{\circ} .(A \oplus B, \Delta)=\left(\Gamma_{1}, \Gamma_{2}\right)$. Then $\Gamma_{1}=A \oplus B, \Gamma_{2}=\Delta$, and the premises are $A, \Gamma_{3}$ and $B, \Delta$. One derives $A \oplus B,\left(\Delta, \Gamma_{3}\right)$, by $(\mathrm{r}-\oplus 3)$.

$(\mathrm{r}-\oplus 3) .\left(\Gamma_{1}, \Gamma_{2}\right), \Gamma_{3}$ cannot be the conclusion of this rule.

( $\mathrm{r}-\oplus 4)$. Then, $\Gamma_{3}=A \oplus B$, and the premises are $\Gamma_{2}, A$ and $\Gamma_{1}, B$. One derives $\Gamma_{1},\left(\Gamma_{2}, A \oplus B\right)$, by $(\mathrm{r}-\oplus 1)$.

Corollary 1. For any sequent $\Gamma, \vdash \Gamma$ if and only if $\vdash_{0} \Gamma$.

We need two new rules.

$$
\left(\mathrm{r}^{\sim} \sim\right) \frac{A, \Gamma}{\Gamma, A^{\sim \sim}} \quad\left(\mathrm{r}^{---}\right) \frac{\Gamma, A}{A^{--}, \Gamma}
$$

Lemma 3. The rules $\left(r_{-} \sim\right)$ and $\left(r_{-}^{--}\right)$are admissible in $S$-InNL.

Proof: By Corollary 1, it suffices to prove that these rules are admissible in $S_{0}$. We give a proof for $\left(\mathrm{r}^{\sim} \sim\right)$. The proof for $\left(\mathrm{r}^{--}\right)$is similar. We prove: if $\vdash_{0} D, \Theta$ then $\vdash_{0} \Theta, D^{\sim \sim}$, by the outer induction on the number of connectives in $D$ and the inner induction on the proof of $D, \Theta$ in $S_{0}$.

Assuming our claim for all $D^{\prime}$ having less connectives than $D$, we run the inner induction.

If $D, \Theta$ is an axiom (id), where $D=p^{(n)}, \Theta=p^{(n+1)}$, then $\Theta, D^{\sim \sim}$ equals $p^{(n+1)} \cdot p^{(n+2)}$, which is an axiom, too. 
Assume that $D, \Theta$ is a conclusion of a rule. If $D$ is not the active formula of the rule (this may only happen for $(\mathrm{r}-\otimes),(\mathrm{r}-\oplus 1),(\mathrm{r}-\oplus 2))$, then one applies the (inner) induction hypothesis to the premise which contains $D$ (this must be $\Gamma[B]$ in $(\mathrm{r}-\oplus 1)$ and $\Gamma[A]$ in $(\mathrm{r}-\oplus 2))$, next applies the same rule. Assume that $D$ is the active formula of the rule. This may happen for $(\mathrm{r}-\otimes)$ and $(\mathrm{r}-\oplus 3)$.

$(\mathrm{r}-\otimes)$. So $D=A \otimes B$ and the premise is $(A, B), \Theta$. By Lemma 2 and the outer induction hypothesis, we obtain $\vdash_{0} A,(B, \Theta), \vdash_{0}(B, \Theta), A^{\sim \sim}$, $\vdash_{0} B,\left(\Theta, A^{\sim \sim}\right), \vdash_{0}\left(\Theta, A^{\sim \sim}\right), B^{\sim \sim}, \vdash_{0} \Theta,\left(A^{\sim \sim}, B^{\sim \sim}\right)$, hence $\vdash_{0} \Theta, D^{\sim \sim}$, by $(\mathrm{r}-\otimes)$.

(r- $\oplus 3)$. So $D=A \oplus B, \Theta=(\Delta, \Gamma)$, and the premises are $A, \Gamma$ and $B, \Delta$. By the (inner or outer) induction hypothesis, $\vdash_{0} \Gamma, A^{\sim \sim}$ and $\vdash_{0} \Delta, B^{\sim \sim}$, hence $\vdash_{0} \Theta, D^{\sim \sim}$, by $(\mathrm{r}-\oplus 4)$.

As a consequence, $\vdash A^{-}, \Gamma$ if and only if $\vdash \Gamma, A^{\sim}$; we write $\Gamma \Rightarrow A$ for $A^{-}, \Gamma$.

TheOREM 1. The cut rules are admissible in $S$-InNL.

Proof: By Corollary 1, it suffices to prove that $\left(\mathrm{cut}^{\sim}\right)$, (cut $\left.{ }^{-}\right)$are admissible in $S_{0}$, this means:

Claim 1. if $\vdash_{0} \Theta[C]$ and $\vdash_{0} \Psi, C^{\sim}$ then $\vdash_{0} \Theta[\Psi]$,

Claim 2. if $\vdash_{0} \Theta[C]$ and $\vdash_{0} C^{-}, \Psi$ then $\vdash_{0} \Theta[\Psi]$.

Claim 2 follows from Claim 1, by Corollary 1 and Lemma 3. We prove Claim 1 by the outer induction on the number of connectives in $C$, the intermediate induction on the proof of $\Theta[C]$ (the major premise), and the inner induction on the proof of $\Psi, C^{\sim}$ (the minor premise). Observe that $A, A^{\sim}$ and $A^{-}$have the same number of connectives.

Let $C=p^{(n)}$. We run the intermediate induction. Let $\Theta\left[p^{(n)}\right]$ be an axiom. If the axiom is $p^{(n)}, p^{(n+1)}$, then $\Psi, C^{\sim}$ equals $\Psi, p^{(n+1)}$, hence it equals $\Theta[\Psi]$. If the axiom is $p^{(n-1)}, p^{(n)}$, then $\Psi, C^{\sim}$ equals $\Psi, p^{(n+1)}$, which yields $\Theta[\Psi]$, by $\left(\mathrm{r}^{--}\right)$. Let $\Theta[C]$ be obtained by a rule. Since $C$ is not the active formula of any rule, then $C$ must occur in one premise of this rule. One applies the induction hypothesis to this premise and $\Psi, C^{\sim}$, then the same rule.

Let $C$ be not an atom. We run the intermediate induction. $\Theta[C]$ is not an axiom, hence it is obtained by a rule. If $C$ is not the active formula of the rule, then we proceed as above. Otherwise we consider several cases, corresponding to particular rules. 
$(\mathrm{r}-\otimes)$. So $C=A \otimes B, C^{\sim}=B^{\sim} \oplus A^{\sim}$, and the premise is $\Theta[(A, B)]$. We run the inner induction. $\Psi, C^{\sim}$ is not an axiom, hence it is obtained by a rule. The only possible rules are $(\mathrm{r}-\otimes),(\mathrm{r}-\oplus 1),(\mathrm{r}-\oplus 2),(\mathrm{r}-\oplus 4)$. For $(\mathrm{r}-\otimes)$, we apply the induction hypothesis to $\Theta[C]$ and the premise of this rule, then we apply this rule. For $(\mathrm{r}-\oplus 1)$, the active bunch introduced by the rule must appear in $\Psi$, hence $\Psi=\Psi^{\prime}[(\Delta, D \oplus E)]$, and the left premise is $\Psi^{\prime}[E], C^{\sim}$. By the induction hypothesis, we obtain $\Theta\left[\Psi^{\prime}[E]\right]$, hence $\Theta[\Psi]$, by $(\mathrm{r}-\oplus 1)$. For $(\mathrm{r}-\oplus 2)$, the argument is similar. For $(\mathrm{r}-\oplus 4), C^{\sim}$ is the active formula, $\Psi=(\Delta, \Gamma)$, and the premises are $\Gamma, B^{\sim}$ and $\Delta, A^{\sim}$. We obtain $\Theta[\Psi]$ by the outer induction hypothesis (applied twice).

$(\mathrm{r}-\oplus 1)$. So $C=A \oplus B, C^{\sim}=B^{\sim} \otimes A^{\sim}, \Theta[C]=\Theta^{\prime}[(\Delta, A \oplus B)]$, and the premises are $\Theta^{\prime}[B]$ and $\Delta, A$. We run the inner induction. $\Psi, C^{\sim}$ is not an axiom, and it can only be obtained by $(\mathrm{r}-\otimes),(\mathrm{r}-\oplus 1),(\mathrm{r}-\oplus 2)$, If $C^{\sim}$ is not the active formula of the rule, then we argue as above. Otherwise, the rule is $(\mathrm{r}-\otimes)$ with the premise $\Psi,\left(B^{\sim}, A^{\sim}\right)$. By (r-shift), we obtain $\left(\Psi, B^{\sim}\right), A^{\sim}$, hence $\Delta,\left(\Psi, B^{\sim}\right)$, by the outer induction hypothesis, then $(\Delta, \Psi), B^{\sim}$, by (r-shift), and finally, $\Theta^{\prime}[(\Delta, \Psi)]$, which equals $\Theta[\Psi]$, by the outer induction hypothesis.

$(\mathrm{r}-\oplus 2) . \quad C$ and $C^{\sim}$ are as above. $\Theta[C]=\Theta^{\prime}[(A \oplus B, \Delta)]$, and the premises are $\Theta^{\prime}[A]$ and $B, \Delta$. We run the inner induction. The only interesting case is that $\Psi, C^{\sim}$ arises by $(\mathrm{r}-\otimes)$ with the premise $\Psi,\left(B^{\sim}, A^{\sim}\right)$. Using (r-shift), ( $\left.\mathrm{r}^{--}\right)$, we obtain: $\left(\Psi, B^{\sim}\right), A^{\sim}$, then $A^{-},\left(\Psi, B^{\sim}\right)$, and $\left(A^{-}, \Psi\right), B^{\sim}$. Hence $\left(A^{-}, \Psi\right), \Delta$, by the outer induction hypothesis, which yields $A^{-},(\Psi, \Delta)$, then $(\Psi, \Delta), A^{\sim}$ (by $(\mathrm{r}-\sim \sim)$ ), and $\Theta^{\prime}[(\Psi, \Delta)]$, by the outer induction hypothesis.

(r- $\oplus 3) . \quad C$ and $C^{\sim}$ are as above, $\Theta[C]=(A \oplus B,(\Delta, \Gamma))$, and the premises are $A, \Gamma$ and $B, \Delta$. We run the inner induction. $\Psi, C^{\sim}$ can be obtained by $(\mathrm{r}-\otimes),(\mathrm{r}-\oplus 1),(\mathrm{r}-\oplus 2)$. We only consider $(\mathrm{r}-\otimes)$ with the active formula $C^{\sim}$ and the premise $\Psi,\left(B^{\sim}, A^{\sim}\right)$. By (r-shift) and the outer induction hypothesis, we obtain $\left(\Psi, B^{\sim}\right), A^{\sim}$, hence $\left(\Psi, B^{\sim}\right), \Gamma$, then $\Psi,\left(B^{\sim}, \Gamma\right)$. From $B, \Delta$ we obtain $\Delta, B^{\sim \sim}$, by $\left(\mathrm{r}^{\sim \sim}\right)$. This yields $\Psi,(\Delta, \Gamma)$, which equals $\Theta[\Psi]$, by the outer induction hypothesis.

$(\mathrm{r}-\oplus 4) . \quad C$ and $C^{\sim}$ are as above. $\Theta[C]=((\Delta, \Gamma), A \oplus B)$, and the premises are $\Gamma, A$ and $\Delta, B$. We run the inner induction. We only consider $(\mathrm{r}-\otimes)$ with the premise $\Psi,\left(B^{\sim}, A^{\sim}\right)$. As above, we obtain: $\left(\Psi, B^{\sim}\right), A^{\sim}$, then $A^{-},\left(\Psi, B^{\sim}\right)$, and $\left(A^{-}, \Psi\right), B^{\sim}$. Hence $\Delta,\left(A^{-}, \Psi\right)$, by the outer induction hypothesis, which yields $\left(\Delta, A^{-}\right), \Psi$, and $(\Delta, \Gamma), \Psi$, by the outer induction hypothesis, since $\Gamma, A$ equals $\Gamma, A^{-\sim}$. 
Observe that $\left(\mathrm{r}^{-} \sim\right),\left(\mathrm{r}^{--}\right)$are derivable in S-InNL with the cut rules. We derive (r- $\sim)$. Assume $A, \Gamma$, which equals $A^{\sim-}, \Gamma . A^{\sim}, A^{\sim \sim}$ is provable, by Lemma 1 . This yields $\Gamma, A^{\sim \sim}$, by $\left(\right.$ cut $\left.^{-}\right)$.

A sequent $\Gamma$ is said to be valid in InNL, if $\mathbf{M}, \mu \models \Gamma$ for all involutive residuated groupoids $\mathbf{M}$ and all valuations $\mu$ in $\mathbf{M}$. We say that a set of sequents $X$ entails a sequent $\Gamma$ in InNL, if for any involutive residuated gropoid $\mathbf{M}$ and any valuation $\mu$ in $\mathbf{M}$, if $\mathbf{M}, \mu \models X$ then $\mathbf{M}, \mu \models \Gamma$. The strong completeness means: $\Gamma$ is provable from $X$ if and only if $X$ entails $\Gamma$, for all $X, \Gamma$. The weak completeness means: $\Gamma$ is provable iff $\Gamma$ is valid.

For any bunch $\Gamma$ we define a formula $f(\Gamma)$ as follows: $f(A)=A$, $f((\Gamma, \Delta))=f(\Gamma) \otimes f(\Delta)$. We have $\mu(\Gamma)=\mu(f(\Gamma))$, for any valuation $\mu$. Since $(\mathrm{r}-\otimes)$ is reversible in S-InNL (also with the cut rules), then sequents $\Gamma, \Delta$ and $f(\Gamma), f(\Delta)$ are deductively equivalent. We assume that all sequents in $X$ are of the form $(A, B)$.

THEOREM 2. S-InNL with (cut $\left.{ }^{\sim}\right)$, (cut ${ }^{-}$) is strongly complete with respect to involutive residuated groupoids.

PROOF: Soundness is easy: the axioms (id) are valid and all rules preserve the truth for $\mu$ in $\mathbf{M}$.

For completeness, we define: $A \leq B$ iff $X \vdash A \Rightarrow B$ (now $\vdash$ denotes the provability in S-InNL with the cut rules). Also: $A \sim B$ iff $A \leq B$ and $B \leq A$. One shows that $\sim$ is a congruence in the algebra of formulas. Also: if $A \sim B$ then $A^{\sim} \sim B^{\sim}$ and $A^{-} \sim B^{-}$. The quotient algebra (with the quotient ordering) is an involutive residuated groupoid. We define: $\mu(p)=$ $[p]_{\sim}$ for $p=p^{(0)}, \mu\left(p^{(n+1)}\right)=\mu\left(p^{(n)}\right)^{\sim}$ for $n \geq 0$, and $\mu\left(p^{(n-1)}\right)=\mu\left(p^{(n)}\right)^{-}$ for $n \leq 0$. Then, $\mu$ satisfies $\mu\left(p^{(n+1)}\right)=\mu\left(p^{(n)}\right)^{\sim}$ for any $n \in \mathbb{Z}$. One easily proves: $\mu(A)=[A]_{\sim}$ for any formula $A$, hence $\mu(\Gamma)=[f(\Gamma)]_{\sim}$ for any bunch $\Gamma$. Consequently, all sequents from $X$ are true for $\mu$. Also: if $X \forall \Gamma, \Delta$, then $\mu(\Gamma) \not \leq \mu(\Delta)^{-}$, hence $\Gamma, \Delta$ is not true for $\mu$.

Corollary 2. The cut-free $S$-InNL is weakly complete with respect to involutive residuated groupoids.

The sequent system S-InNL1 is an extension of S-InNL. We add the empty bunch $\epsilon$ and constants 1 and 0 . Sequents are all nonempty bunches. We add one new axiom and two new rules.

$$
\text { (a.0) } 0 \quad(\mathrm{r}-1) \frac{\Gamma[\Delta]}{\Gamma[(1, \Delta)]} \frac{\Gamma[\Delta]}{\Gamma[(\Delta, 1)]}
$$

In rules $(\mathrm{r}-\oplus 1),(\mathrm{r}-\oplus 2)$ we admit $\Delta=\epsilon$, and similarly for $\left(\right.$ cut $\left.^{\sim}\right),\left(\right.$ cut $\left.^{-}\right)$. 
In the derivable rules $(\mathrm{r}-\oplus 3),(\mathrm{r}-\oplus 4)$ both $\Gamma$ and $\Delta$ may be empty; in rules $\left(\mathrm{r}^{\sim} \sim\right),\left(\mathrm{r}^{--}\right) \Gamma$ may be empty. We extend metalanguage negations by: $1^{\sim}=1^{-}=0$ and $0^{\sim}=0^{-}=1$.

The algebraic models are unital involutive residuated monoids. We define: $\mathbf{M}, \mu \models \Gamma$ iff $\mu(\Gamma) \leq 0$. Also $\mu(\epsilon)=\mu(1)=1$.

All results, proved above and appropriately modified, remain true for S-InNL1. The proofs are quite similar. For Lemma 1, observe that $1^{-}, 1$ equals $0,0^{\sim}$ and $1,1^{\sim}$ equals $0^{-}, 0$; they are obtained from (a.0) by (r-1). $S_{0}$ is defined in the same way as above (in relation to S-InNL1). In Lemma 2 , (r-shift) is considered for nonempty $\Gamma_{i}$ only. The proofs of Lemma 2, Lemma 3 and Theorem 1 require more cases. For instance $\left(\Gamma_{1}, \Gamma_{2}\right), \Gamma_{3}$ can be obtained by $(\mathrm{r}-\oplus 1)$ with premises $B$ and $\left(\Gamma_{1}, \Gamma_{2}\right), A$; then $\Gamma_{3}=A \oplus B$. We obtain $\Gamma_{1},\left(\Gamma_{2}, A\right)$, by the induction hypothesis, and apply $(\mathrm{r}-\oplus 2)$. We leave further changes to the reader. In the proof of Theorem 2 we may assume that all sequents in $X$ are single formulas. We define $f(\epsilon)=1$.

Observe that from $A, A^{\sim}$ one obtains $A \otimes A^{\sim}$ in S-InNL1, but not in S-InNL. In S-InNL1 $A, A^{\sim}$ expresses the algebraic law $a \otimes a^{\sim} \leq 0$, while in S-InNL it expresses $a \leq a$.

These systems can be augmented with additive connectives $\wedge, \vee$, interpreted as meet and join in lattice-ordered (l.o.) involutive residuated groupoids. The rules for them are as follows.

$$
(\mathrm{r}-\wedge) \frac{\Gamma\left[A_{i}\right]}{\Gamma\left[A_{1} \wedge A_{2}\right]}(i \in\{1,2\}) \quad(\mathrm{r}-\vee) \frac{\Gamma[A] \quad \Gamma[B]}{\Gamma[A \vee B]}
$$

The resulting logics may be denoted by InFNL, InFNL1, since FNL (Full $\mathrm{NL}$ ) is used for NL with $\wedge, \vee$, and the sequent systems by S-InFNL, S-InFNL1. InFNL1 is equivalent to InGL in [7]. All results of this section (appropriately modified) are true for InFNL and InFNL1. Our system S-InFNL1 is simpler than the two-sided sequent system for InGL, proposed in [7]. Using S-InFNL1, one may simplify certain proofs of model-theoretic results, e.g. the finite model property [7].

\section{PTIME complexity}

Let $T$ be a set of formulas. By a $T$-sequent we mean a sequent built from formulas from $T$. By a $T$-proof we mean a proof consisting of $T$-sequents. We write $\vdash^{T} \Gamma$ (resp. $\vdash_{0}^{T} \Gamma$ ), if there exists a $T$-proof of $\Gamma$ in S-InNL (resp. $\left.S_{0}\right)$. S-InNL and $S_{0}$ possess the subformula property: if $\vdash \Gamma$ then $\vdash^{T} \Gamma$, 
where $T$ is the subformula closure of the set of formulas in $\Gamma$, and similarly for $\vdash_{0}$.

We prove the PTIME complexity of InNL in the following way. A sequent is said to be restricted, if it contains at most three formulas. So the restricted sequents are of the form $(A, B),((A, B), C)$ or $(A,(B, C))$. We show that a restricted sequent $\Gamma$ is provable in S-InNL if and only if $\Gamma$ is provable in S-InNL limited to restricted $c(T)$-sequents, where $c(T)$ is a finite superset of the set $T$, consisting of all formulas in $\Gamma$. The set $c(T)$ is computable in time polynomial in the size of $\Gamma$. The number of restricted $c(T)$-sequents is $O\left(n^{3}\right)$, where $n$ is the cardinality of $c(T)$, and all provable restricted $T$-sequents can be computed in polynomial time. $\Gamma, \Delta$ is provable if and only if $f(\Gamma), f(\Delta)$ is provable, and the latter sequent is restricted.

We define $T^{\sim}=\left\{A^{\sim}: A \in T\right\}, T^{-}=\left\{A^{-}: A \in T\right\}$, and $c(T)=$ $T \cup T^{\sim} \cup T^{-}$. Let $T$ be a finite set of formulas, closed under subformulas. We define an auxiliary system $S_{1}^{T}$ (we often write $S_{1}$ ) as follows. The axioms are all sequents $A^{-}, A$ and $A, A^{\sim}$, for $A \in T$. The inference rules are all rules of $S_{0}$, limited to restricted $c(T)$-sequents with the active formula in $T$ and $\left(\right.$ cut $\left.^{\sim}\right)$, $\left(\right.$ cut $\left.^{-}\right)$, limited to $c(T)$-sequents. By $\vdash_{1}$ we denote the provability in $S_{1}$. We show that $S_{1}$ possesses an interpolation property.

Lemma 4. If $\vdash_{1} \Theta[\Psi], \Theta[\Psi] \neq \Psi$, then there exists $D \in c(T)$ such that $\vdash_{1} \Theta[D]$ and either $\vdash_{1} D^{-}, \Psi$ or $\vdash_{1} \Psi, D^{\sim}$.

Proof: We proceed by induction on proofs of $\Theta[\Psi]$ in $S_{1}$. If $\Psi$ is a formula, say $\Psi=A$, we put $D=A$. Clearly $\vdash_{1} \Theta[D]$. If $A \in T$, then $\Psi, A^{\sim}$ is an axiom. If $A \in T^{-}$, then $A^{\sim} \in T$, hence $\Psi, A^{\sim}$ is the axiom $A^{\sim-}, A^{\sim}$. If $A \in T^{\sim}$, then $A^{-} \in T$, hence $A^{-}, \Psi$ is the axiom $A^{-}, A^{-\sim}$.

We assume that $\Psi$ is not a formula. So $\Theta[\Psi]$ cannot be an axiom. We consider several cases corresponding to the rules of $S_{1}$.

$(\mathrm{r}-\otimes)$. The premise is a restricted sequent. The only possibilities are:

$$
\frac{(A, B), C}{A \otimes B, C} \quad \frac{C,(A, B)}{C, A \otimes B}
$$

In both cases a bunch properly contained in the conclusion must be a formula. 
$(\mathrm{r}-\oplus 1)$. The only possibilities are:

$$
\frac{C_{1}, B C_{2}, A}{C_{1},\left(C_{2}, A \oplus B\right)} \quad \frac{B, C_{1} C_{2}, A}{\left(C_{2}, A \oplus B\right), C_{1}} .
$$

So $\Psi=\left(C_{2}, A \oplus B\right)$. We put $D=B$. Then $\Theta[D]$ equals the first premise. $B \in T$, hence $B, B^{\sim}$ is an axiom, and we obtain $\left(C_{2}, A \oplus B\right), B^{\sim}$ from this axiom and the second premise, by $(\mathrm{r}-\oplus 1)$. For $(\mathrm{r}-\oplus 2)$ the argument is symmetrical (take $D=A$ and use the axiom $A^{-}, A$ ).

$(\mathrm{r}-\oplus 3)$. The only possibility is:

$$
\frac{A, C_{1} B, C_{2}}{A \oplus B,\left(C_{2}, C_{1}\right)} .
$$

So $\Psi=\left(C_{2}, C_{1}\right)$. $A \oplus B \in T$, and we put $D=(A \oplus B)^{\sim}$. Then $\Theta[D]$ is the axiom $A \oplus B,(A \oplus B)^{\sim}$ and $D^{-}, \Psi$ is the conclusion.

$(\mathrm{r}-\oplus 4)$. The only possibility is:

$$
\frac{C_{1}, A C_{2}, B}{\left(C_{2}, C_{1}\right), A \oplus B} .
$$

So $\Psi=\left(C_{2}, C_{1}\right) . A \oplus B \in T$, and we put $D=(A \oplus B)^{-}$. Then $\Theta[D]$ is the axiom $(A \oplus B)^{-}, A \oplus B$ and $\Psi, D^{\sim}$ is the conclusion.

(cut $\sim$ ). So $\Theta[\Psi]=\Gamma[\Delta]$ and the premises are: $\Gamma[A]$ and $\Delta, A^{\sim}$. If $\Psi$ occurs in $\Gamma[]$, then we take $D$ for $\Psi$ in $\Gamma[A]$ ( $\Psi$ cannot contain $A)$. We obtain $\Theta[D]$, by (cut $\sim$ ). If $\Psi$ occurs in $\Delta$, then we take $D$ for $\Psi$ in $\Delta, A^{\sim}$. We obtain $\Theta[D]$ as above. The last possibility is: $\Gamma[\Delta]=\Gamma_{1}\left[\Gamma_{2}[\Delta]\right]$ and $\Psi=\Gamma_{2}[\Delta], \Psi \neq \Delta$. Then, $\Gamma[A]=\Gamma_{1}\left[\Gamma_{2}[A]\right]$. Since $\Psi \neq \Gamma[\Delta]$, then $\Gamma_{2}[A] \neq \Gamma[A]$. We take $D$ for $\Gamma_{2}[A]$ in $\Gamma[A]$. We have: $\vdash_{1} \Gamma_{1}[D]$ and either $\vdash_{1} D^{-}, \Gamma_{2}[A]$, or $\vdash_{1} \Gamma_{2}[A], D^{\sim}$. We obtain $\vdash_{1} D^{-}, \Gamma_{2}[\Delta]$ or $\vdash_{1} \Gamma_{2}[\Delta], D^{\sim}$, by $\left(\right.$ cut $\left.^{\sim}\right)$. For $\left(\right.$ cut $\left.^{-}\right)$the argument is similar.

For a sequent $\Gamma$ we take $T$ as the subformula closure of the set of all formulas appearing in $\Gamma$. We define $S_{1}^{T}$ as above.

Lemma 5. $\Gamma$ is provable in $S$-InNL if and only if $\vdash_{1} \Gamma$.

Proof: The 'if' part follows from the fact that the axioms of $S_{1}$ are provable in S-InNL and the rules of $S_{1}$ either are instances of rules of S-InNL, or are derivable in S-InNL. We prove the only-if part.

Assume that $\Gamma$ is provable in S-InNL. By Corollary 1, $\Gamma$ is provable in $S_{0}$. By the subformula property, $\vdash_{0}^{T} \Gamma$. It suffices to show that all 
axiomatic $T$-sequents of $S_{0}$ are provable in $S_{1}$ and all rules of $S_{0}$, limited to $T$-sequents, are admissible in $S_{1}$.

If $p^{(n)} \in T$, then (id) is an axiom $A, A^{\sim}$ of $S_{1}$.

$(\mathrm{r}-\otimes)$. Assume that $\vdash_{1} \Gamma[(A, B)]$ and $\Gamma[A \otimes B]$ is a $T$-sequent. So $\Gamma[(A, B)]$ is different from $(A, B)$. By Lemma 4 , there exists $D \in c(T)$ such that $\vdash_{1} \Gamma[D]$ and either $\vdash_{1} D^{-},(A, B)$ or $\vdash_{1}(A, B), D^{\sim}$. By $(\mathrm{r}-\oplus)$ in $S_{1}$, we obtain $\vdash_{1} D^{-}, A \otimes B$ or $\vdash_{1} A \otimes B, D^{\sim}$. Consequently, $\vdash_{1} \Gamma[A \otimes B]$, by $\left(\right.$ cut $\left.^{-}\right)$or $\left(\right.$cut $\left.^{\sim}\right)$.

(r- $\oplus 1)$. Assume that $\vdash_{1} \Gamma[B], \vdash_{1} \Delta, A$ and $\Gamma[(\Delta, A \oplus B)]$ is a $T$-sequent. By Lemma 4, there exists $D \in c(T)$ such that $\vdash_{1}, D, A$ and either $\vdash_{1}$ $D^{-}, \Delta$ or $\vdash_{1} \Delta, D^{\sim}$. From $D, A$ and $B, B^{\sim}$ (an axiom of $S_{1}$ ) we obtain $(D, A \oplus B), B^{\sim}$, by $(\mathrm{r}-\oplus 1)$ in $S_{1}$, hence $(\Delta, A \oplus B), B^{\sim}$ by a cut rule in $S_{1}$. This yields $\Gamma[(\Delta, A \oplus B)]$ by $\left(\right.$ cut $\left.^{\sim}\right)$ in $S_{1}$. For $(\mathrm{r}-\oplus 2)$ the argument is similar.

(r- $\oplus 3)$. Assume that $\vdash_{1} A, \Gamma, \vdash_{1} B, \Delta$ and $A \oplus B,(\Delta, \Gamma)$ is a $T$-sequent. By Lemma 4 , there exist $D_{1} \in T$ and $D_{2} \in T$ such that $\vdash_{1} A, D_{1}, \vdash_{1} B, D_{2}$, either $\vdash_{1} D_{1}^{-}, \Gamma$ or $\vdash_{1} \Gamma, D_{1}^{\sim}$, and either $\vdash_{1} D_{2}^{-}, \Delta$ or $\vdash_{1} \Delta, D_{2}^{\sim}$. We obtain $A \oplus B,\left(D_{2}, D_{1}\right)$, by $(\mathrm{r}-\oplus 3)$ in $S_{1}$, hence $A \oplus B,(\Delta, \Gamma)$ by the cut rules in $S_{1}$. For $(\mathrm{r}-\oplus 4)$ the argument is similar.

For any rule of $S_{1}$, if the conclusion is restricted, then the premises are restricted. This holds for $\otimes-$ and $\oplus$-rules, by the definition of $S_{1}$, and is obvious for the cut rules. Therefore every restricted sequent $\Gamma$ provable in S-InNL has a proof in $S_{1}^{T}$ which consists of restricted $c(T)$-sequents only ( $T$ is constructed from $\Gamma$, as above).

We want to verify the provability of a restricted sequent $\Gamma$. We define the size of $\Gamma(s(\Gamma))$ as follows: $s\left(p^{(n)}\right)=|n|+1, s(A \circ B)=s(A)+$ $s(B)+1$, for $\circ \in\{\otimes, \oplus\}, s\left(\left(\Gamma_{1}, \Gamma_{2}\right)\right)=s\left(\Gamma_{1}\right)+s\left(\Gamma_{2}\right)$. Here $|n|$ can be understood as the absolute value of $n$ or as the length of the binary or decimal representation of $n$. Let $n=s(\Gamma)$. We compute $T$ in $O\left(n^{2}\right)$ and $T$ has at most $n$ elements. We compute $c(T)$ in $O(n)$ and $c(T)$ has at most $3 n$ elements. The number of all possible restricted $c(T)$-sequents is $O\left(n^{3}\right)$. Those which are provable in $S_{1}^{T}$ can be computed in time polynomial in $n$. The procedure can be described as follows.

We compute a list $\Gamma_{1}, \Gamma_{2}, \Gamma_{3}, \ldots$ of all restricted $c(T)$-sequents provable in $S_{1}^{T}$. First, we write all axioms, say $\Gamma_{1}, \ldots, \Gamma_{k}$. They cannot be premises of $(\mathrm{r}-\otimes)$. For $i=2,3,4, \ldots$, we compute all possible conclusions of the $\oplus$-rules and cut rules applied to $\Gamma_{i}$ and $\Gamma_{j}$, for any $j<i$, and add 
them to the list (we only add new sequents). For any $i$, this can be done in time $O(n i)$, assuming that one rule is executed in time $O(n)$. For $i>k$, one also admits $(\mathrm{r}-\otimes)$, if applicable to $\Gamma_{i}$. Since the complete list has at most $O\left(n^{3}\right)$ elements, we obtain a rough estimation $O\left(n^{7}\right)$.

Theorem 3. InNL is PTIME.

This theorem remains true for InNL1. Now, restricted sequents may be single formulas. In the cut rules, the conclusion can be shorter than the major premise, if $\Delta=\epsilon$. In $S_{1}$ for S-InNL1, we admit the cut rules with $\Delta \neq \epsilon$ only. Lemma 4 is proved for $\Psi \neq \epsilon$. We leave further details to the reader.

With $\wedge, \vee$, these logics are PSPACE; the lower bound of complexity is not known. Associative versions of these logics without additives are $N P$-complete [16] and with additives PSPACE-complete. The latter logics are evidently PSPACE. They are PSPACE-hard, since they contain Full Lambek Calculus and are contained in MALL; see [4].

\section{References}

[1] V. M. Abrusci, Phase semantics and sequent calculus for pure noncommutative classical linear propositional logic, Journal of Symbolic Logic 56 (1991), pp. 1403-1451.

[2] A. Bastenhof, Categorial Symmetry, Ph.D. Thesis, University of Utrecht (2013).

[3] W. Buszkowski, Lambek Calculus with Nonlogical Axioms, [in:] Casadio, C., Scott, P.J. and Seely, R.A.S., Language and Grammar. Studies in Mathematical Linguistics and Natural Language. CSLI Lecture Notes 168 (2005), pp. 77-93.

[4] W. Buszkowski, An Interpretation of Full Lambek Calculus in Its Variant without Empty Antecedents of Sequents, [in:] Asher, N. and Soloviev, S. (eds.), Logical Aspects of Computational Linguistics, Lecture Notes in Computer Science, vol. 8535 (2014), Springer, pp. 30-43.

[5] W. Buszkowski, On Classical Nonassociative Lambek Calculus, [in:] Amblard, M,, de Groote, Ph., Pogodalla, S., and Retoré, C. (eds.), Logical Aspects of Computational Linguistics, Lecture Notes in Computer Science, vol. 10054 (2016), Springer, pp. 68-84. 
[6] W. Buszkowski, Phase Spaces for Involutive Nonassociative Lambek Calculus, [in:] Loukanova, R. and Liefke, K. (eds.), Proc. Logic and Algorithms in Computational Linguistics (LACompLing 2017), Stockholm University, (2017), pp. 21-45.

[7] N. Galatos and P. Jipsen, Residuated frames with applications to decidability, Transactions of American Mathematical Society 365 (2013), pp. 1219-1249.

[8] N. Galatos, P. Jipsen, T. Kowalski and H. Ono, Residuated Lattices. An Algebraic Glimpse at Substructural Logics, Elsevier (2007).

[9] J.-Y. Girard, Linear logic, Theoretical Computer Science 50 (1987), pp. $1-102$.

[10] Ph. de Groote and F. Lamarche, Classical Non-Associative Lambek Calculus, Studia Logica $71 / 3$ (2002), pp. 355-388.

[11] J. Lambek, On the calculus of syntactic types, [in:] Jakobson, R. (ed.) Structure of Language and Its Mathematical Aspects, pp. 166-178. AMS, Providence (1961).

[12] J. Lambek, Cut elimination for classical bilinear logic, Fundamenta Informaticae 22 (1995), pp. 53-67.

[13] J. Lambek, Some lattice models of bilinear logic, Algebra Universalis 34 (1995), pp. 541-550.

[14] G. Malinowski, Many-valued Logics, Oxford Logic Guides 25, The Clarendon Press, Oxford University Press, 1993.

[15] M. Okada and K. Terui, The finite model property for various fragments of intuitionistic linear logic, Journal of Symbolic Logic 64 (1999), pp. 790-802.

[16] M. Pentus, Lambek calculuc is NP-complete, Theoretical Computer Science 357 (2006), pp. 186-201.

Faculty of Mathematics and Computer Science

Adam Mickiewicz University

Poznań, Poland

e-mail: buszko@amu.edu.pl 